\title{
Effects of temporal variation of auxiliary light stimuli upon detectability of tonal signals'
}

WILLIAM H, WATKINS ${ }^{2}$ AND JOHN R. SCHJELDERUP USAF DECISION SCIENCES LABORATORY, BEDFORD, MASS.

Literature is surveyed briefly which reported the effect upon hearing of varying the onset of a light relative to the onset of a tone. Four forced-choice experiments involving approximately 32,000 trials of the four-altemative type are reported whose results show both similarities and differences when compared with earlier experiments which did not use forced-choice. Tentative conclusions derived from all the experiments described also take into account the effect of cessation of the light relative to the tone.

Several studies have demonstrated a dependence of auditory performance upon the onset time of auxiliary light stimuli, relative to onset of the possible acoustic signal. Relevant information provided in three reports of such earlier work will be discussed, followed by accounts of four new experiments.

Auditory psychophysical tasks can be devised with or without controlled variables in other modalities. It is frequently convenient in auditory sensitivity measurement situations to include a visual stimulus, commonly considered as serving a time-specifying function for $S$. Although the issue of the specific function(s) served by photic auxdliaries to auditory tasks is an interesting one, it will not be addressed in this report (see Watkins, 1965, 1966).

Our purpose is, rather, to provide a partial, empirical answer to a practical question: What onset time and cessation properties should be established for a light stimulus, if it is to contribute maximally to auditory efficiency in a tone-detection task?

\section{EARLIER EXPERIMENTS}

Child and Wendt (1938), using a weak 1000 cps tone of $165 \mathrm{msec}$. duration as the auditory stimulus, tested for frequency of correct response (key press) under the conditions of light stimulation indicated in Fig. 1. On a substantial number of trials (80 per S) no tone was presented. "Yes" response data under this catch-trial condition is available (Child \& Wendt, 1938, p. 118), Returning to Fig. 1, notice that clicks were used as cues for listening and reporting times. Except for the catch-trials, tones were sounded at regular intervals of $15 \mathrm{sec}$. Duration of the light stimulus (a $1^{\circ}$ patch at $50 \mathrm{ft} .-\mathrm{L}$ ) was $1 / 10$ sec., i.e., less than that of the tone.

The substance of the results obtained by Child and Wendt is fairly well known: Highest auditory sensitivity appeared for the pooled subject group at a light-onset precedence (re tone) of $.5 \mathrm{sec}$. Various other light conditions were most favorable for
Child \& Woadt (1939)

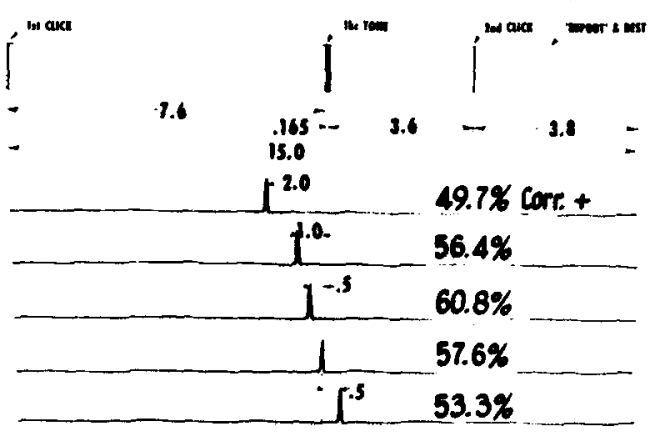

Fig. 1. Trial events with five photic conditions used by Child and Wendt (1938). Horizontal dimensions are seconds.

five of the 11 Ss; zero precedence was the condition averaging second highest.

Egan, Schulman, and Greenberg (1961), in a "memory time" experiment, employed a confidence-rating response type of tone detection task where the 1000 cps tone had .5 probability of occurrence on any trial. The duration of the "alerting light" was twice that of the .25 sec. tone. Figure 2 depicts six of

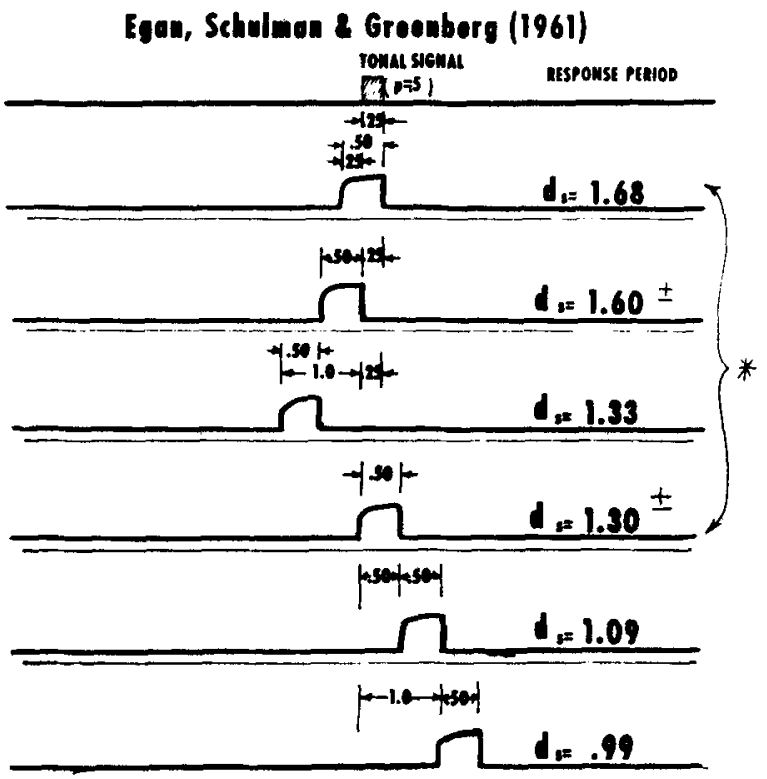

Fig. 2. Composition of trial with six of the photic conditions used by Egan et al, (1961). Detectability indices $\left(d_{s}\right)$ renect auditory efficiency of the $S$ group. Detectability indices under the two conditions identified by the asterisk were reported to differ significantly. Times shown in seconds. 
the Egan et al light conditions. Onset of light 2 sec. after the observation period constituted a seventh condition.

Eight Ss averaged highest detection scores with light precedence of .25 sec., while remaining conditions averaged progressively lower in the order $.5,1.0,0.0,-.5,-1.0$, and $-2.0 \mathrm{sec}$. of light precedence.

Part of an experiment by Treisman (1964) is also pertinent. His signal was a $50 \mathrm{msec}$. intensification of a constant $500 \mathrm{cps}$ tone. Two neon-bulb light stimulus sources were employed. One provided a constant 1 sec. "pre-warning," then an "accessory" light came on, followed after a varied time by the signal. (See Fig. 3). Negative light precedence did not occur in this experiment, as it had in the two experiment previously described. Included were catch trials numbering $1 / 20$ of the total of actual signals. Yes-No response data were reported in terms of percentages correct.

Figure 3 shows Treisman's results. A devlation from monotonicity associated with precedence of 1.25 sec. must be noted, but is not thought to be of importance.

The three studies cited differed in many respects, for example, in auditory signal frequencies and durations, auxiliary light intensities and durations, and psychophysical task situations. Nevertheless, there is much agreement of relevant results. The light precedence time span from 1.0 sec. to synchronous light-tone onset was sampled in all three experiments, and all reflected improvement of detection performance occurring as precedence was reduced, down

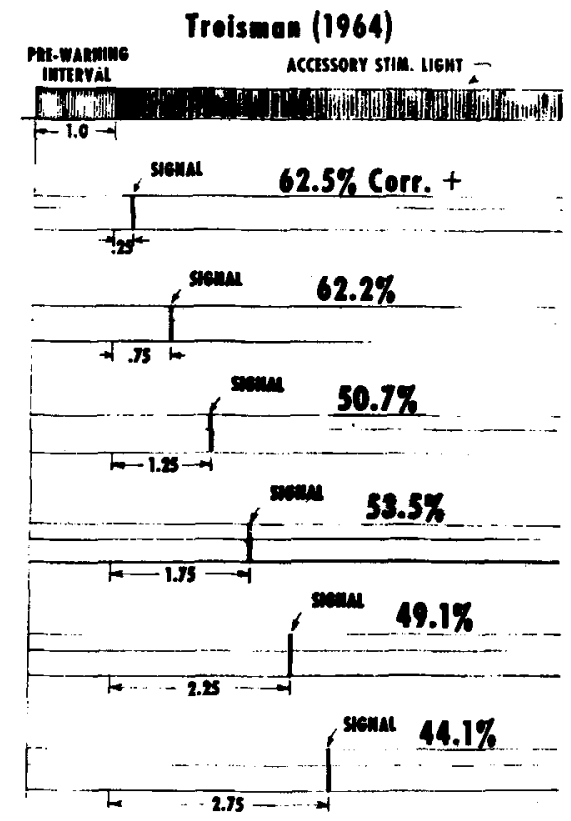

Fig. 3. Trial composition and outcome information for an experiment reported by Treisman (1964). Top bar: photic events. Signal = intensification of $500 \mathrm{cps}$ tone. Times shown in seconds. to precedence of .5 sec. Child and Wendt found synchronous onset scores slightly lower than those for .5 sec. precedence. There was noticeable improvement between .5 and $.25 \mathrm{sec}$. light precedence for Egan et al. Treisman found a minute amount of improvement over the precedence "distance" between .75 and .25 sec.

While synchrony showed only small depression compared to scores at $.5 \mathrm{sec}$. precedence for Chlld and Wendt, the fall in tone detectability between $.25 \mathrm{sec}$. precedence and synchronous onsets was severe in the Egan et al study. A suggestion contained in the latter report is that in the absence of advance warning of an observation interval, having the signal begin with the light may result in listeners being "caught unawares" so that they may "miss" the first part of the signal. The Child and Wendt Ss had heard a click $7.6 \mathrm{sec}$. in advance of all tones, irrespective of light events, and their detection of the tone was little impaired when light precedence was reduced to nil.

There is another factor to be noted in connection with the sharp drop of scores at zero precedence in the Egan et al experiment. That condition involved a carrying over of the light stimulus for $.25 \mathrm{sec}$. beyond cessation of the tonal signal. The highest average resulted under the condition which involved not only light onset precedence of .25 sec. but also concurrent endings of the two stimulus periods. Possible significance of that relation is discussed below.

\section{PRESENT EXPERIMENTS - GENERAL}

The four experiments now to be reported employed a four interval forced choice technique in which every trial included a tonal signal $(1,011 \mathrm{cps})$ presented in one of the intervals. A "ready" light preceded the group of four intervals and a "vote" light followed them. (See top bars of Figs. 4-7). In addition, each experiment employed an auxiliary light which was flashed in some temporal association with each interval (exception: the no-flash condition of Experiment 1). Experiment 1 was unique in that an additional time cue was provided. This was an increase of the level of the white noise background coextensive with the intervals. (See first and second bar of Fig. 4.)

Ss were seated in a chair with arm rests within an anechoic chamber-forehead resting against a padded, curved bar, which established the distance from the eyes to a 1-1/2 in. circular light patch at approximately 43 in. Grason-Stadler Model HD 30 earphones with cushions were worn throughout experimental runs. All acoustic stimuli were presented binaurally. Noise was filtered to 100-6800 cps. Visual stimuli were presented at seated eye level in an unrestricted, darkened field. Ss were instructed to watch the light stimulus patch throughout all tests. All light conditions were represented about equally in the training of each $\mathrm{S}$. 


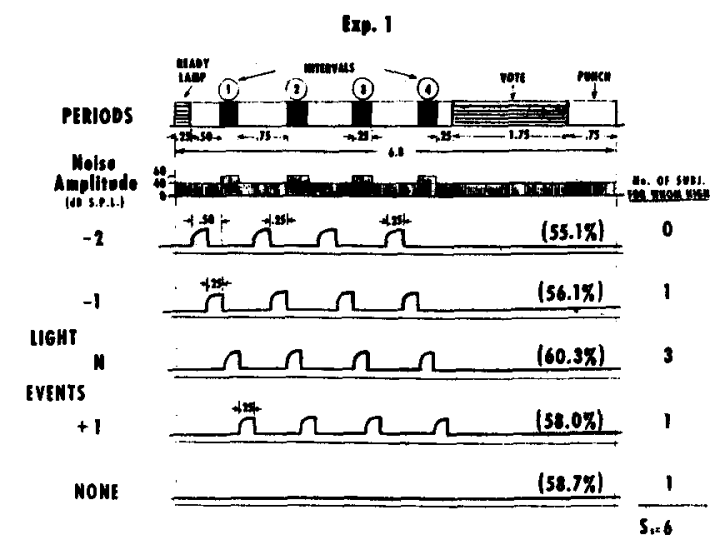

Fig. 4. Trial composition of Experiment 1 with outcome data. Two bars at top contain constant event schedule. The five lower bars describe the various photic conditions. Scores are shown in average percentage of responses correct; times in seconds.

A run consisted of a series (usually numbering 48) of four-alternative forced-choice trials. Before each experiment, instructions concerning the apparatus and the detection and response tasks were given the Ss, usually as a group. Ss were given pre-experiment practice with higher than normal signal levels to familiarize them with the signal to be detected and with the auxiliary lights. In no case was any expected direction of results made known to Ss, although making comparisons between light conditions was an announced intention.

A run began when $\mathrm{S}$ operated a switch at his chair. The first trial was followed automatically (and without delay) by the second, etc., until completion of the run. 3 Four independent blocks of 12 trials comprised the run, with signals distributed equally among the four intervals, for each block. For any $S$, a run sequence was used only once. $S$ responded on each trial by pressing one of four voting keys mounted on his chair in a manner convenient for tactual differentiation. He was informed of his score at the end of each run.

\section{EXPERIMENT 1}

\section{Method}

Figure 4 shows one trial of Experiment 1 with only one of the diagrammed light conditions applicable to any one run. After preliminary practice, each of six adult Ss performed a total of 40 runs; each of the five conditions occurred eight times. Runs for each $\mathrm{S}$ were grouped into five-run sets (one run per condition). The sequence of conditions within sets was varied to minimize the chance of possible effects of order. The visual stimulus was raised from a "fixation" intensity of approximately $4 \mathrm{ft} .-\mathrm{L}$ to a "bright" level of $1350 \mathrm{ft} .-\mathrm{L}$ during four periods within each trial by an automatic switching device. No intensifications were used, of course, for the last condition shown in Fig. 4. A 5/8-in. "ready" lamp situated about 2 in. above the stimulus light was illuminated at about $52 \mathrm{ft}$.- L during the period designated in Fig. 4.

\section{Results and Discussion}

Scores of runs, in terms of percentage of responses correct (forced-choice detection of $.25 \mathrm{sec}$. duration tonal signals) were subjected to analysis of variance in a Conditions by Subjects by Sets matrix. The variables of interest did not show statistically significant differences.

The outcome of Experiment 1 is considered meaningful in spite of the lack of statistical significance. Group means appear to reflect rising detectability of the tonal signals as the light stimuli occurred more nearly in coincidence with possible signal intervals. All light stimuli tended to show inhibitory effects, except for the $\mathrm{N}$ condition which uniquely surpassed the no-flash condition, by about $1-1 / 2$ (in percentage correct average). It was rather unexpected that the +1 condition would average slightly superior to -1 . The latter condition was best for one $S$, and the same was true of +1 and the no-flash conditions.

\section{Method}

\section{EXPERIMENT 2}

In this experiment, the possible signal intervals were reduced to the shortest time available: $.17 \mathrm{sec}$. (See Fig. 5.) Separations between intervals, and all remaining temporal components of trials were maintained at the values employed in Experiment 1. Just two light conditions were used. Flashes in the -1 condition occurred for $1 / 4$ sec., ending in coincidence with the beginning of each interval. In the $\mathrm{N}$ conditicn, the light flash duration was equal to that of the tonal signal, $.17 \mathrm{sec}$. Conditions $\mathrm{N}$ and -1 thus differed both in duration of light flashes and in their onsets with respect to times of possible signals.

Auditory signals in this experiment (and the later ones) were presented against an acoustic background of steady white noise. Six female undergraduates served as Ss, each $S$ performing five runs of each

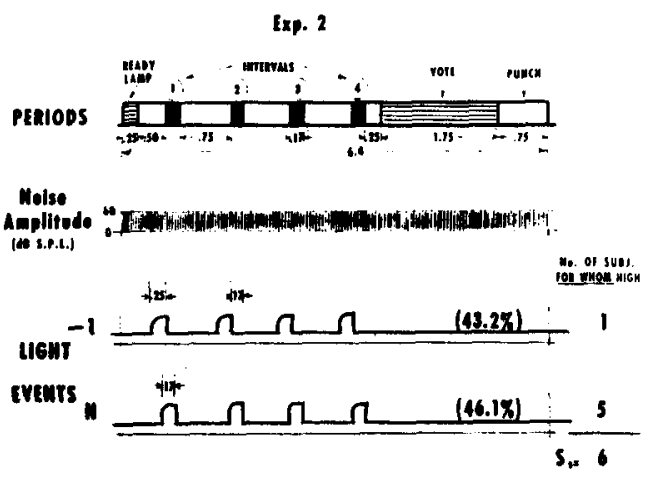

Fig. 5. Conditions and outcome information for Experiment 2 . Scores are in average percentage of responses correct; times in seconds. 


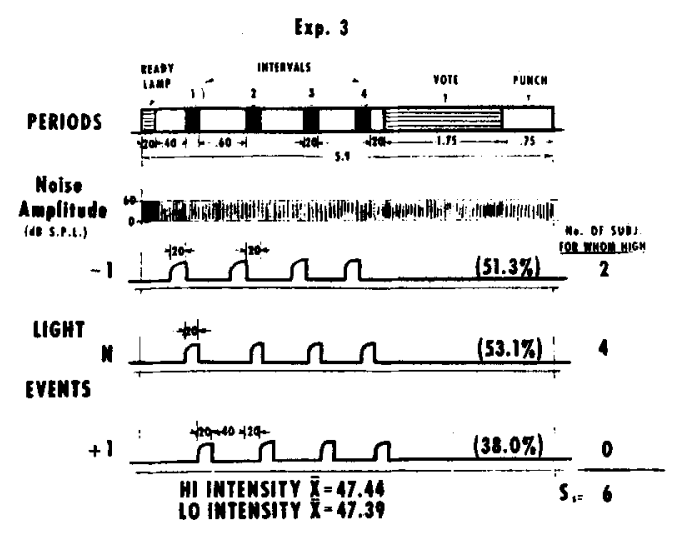

Fig. 6. Conditions and outcome information for Experiment 3.

of the two conditions in sets of two runs. In all other known respects, experimental and environmental conditions were the same as described for Experiment 1.

\section{Results}

Once more, a three-way analysis of variance revealed no significant differences. The average percentage of responses correct was 46.1 under Condition $\mathrm{N}$ and 43.2 under -1 . Five of the six Ss "conformed" to the group trend, although in every case, at least one of the flve sets contained a -1 run superior to its N companion.

\section{Discussion}

Child and Wendt, and Egan et al, the only investigations found possessing comparability, were consistent in finding reduced tone detectability when synchronous onset replaced small amounts of light precedence. We have now presented the results of two experiments in which no such reduction occurred. There was, as noted, a tendency toward improvement accompanying that replacement in our experiments. Ours were forced-choice trials, however, while there was but a single observation period per trial in the other studies mentioned. That diffcrence may account for some of the apparent divergence in results.

\section{EXPERIMENT 3}

\section{Method}

This experiment used the same six Ss as Experiment 2. As shown in Fig. 6, the three light stimulation conditions all involved flashes of equal duration, matching the $.20 \mathrm{sec}$. duration of the intervals. One interval per trial contained a $.20 \mathrm{sec}$. tonal signal. A two-level light intensity variable was included in this experiment in which half the runs for each $S$ were done with flashes of about $740 \mathrm{ft} .-\mathrm{L}$ and half with flashes of $80 \mathrm{ft}$.-L. Dim, or fixation, intensities were only $1 / 200$ of those levels. Six runs constituted a set, and each $\mathrm{S}$ performed five sets. The experiment was like the preceding ones in other respects.

\section{Results}

The light intensity variable was of trivial effect. The mean for high flash intensity was 47.44 (percentage correct responses) and that for the weaker flashes was 47.39. This closeness of high and low intensity data was preserved at all light condition and successive set comparisons. Combining flash intensities and averaging over sets and Ss produced means as follows: -1 : $51.3 ; \mathrm{N}: 53.1 ;+1$ : 38.0. Analyses of variance established the superiority of $N$ over +1 at the .01 confidence level, and that of -1 over +1 at the .05 level. The $\mathrm{N}$ condition advantage over -1 was not significant. Sets, and the several two- and three-way interactions, were likewise not significant variables.

\section{Discussion}

Had Condition +1 not been included, the outcome of Experiment 3 would be accurately described as duplicating the findings of Experiment 2. In Experiment 3 , Condition +1 produced the lowest totals for five of the Ss.

\section{Method}

\section{EXPERIMENT 4}

Duration of light stimuli was incorporated as a variable for this experiment. Figure 7 shows the four light conditions employed. One duration, .4 sec., was duplicated in Condition $-1, \mathrm{~N}$ (onset time .2 sec. before intervals) and in $\mathrm{N},+1$ (onset time coincident with start of intervals). Four runs comprised a set; six Ss who were not involved in the previous experiments performed elght sets each. Apparatus and procedures were, otherwise, the same as those of Experiment 3, with light flash intensity $740 \mathrm{ft}$. $-\mathrm{L}$.

\section{Results and Discussion}

The four condition averages fell evenly into high $(-1, N$ and $-1, N,+1)$ and low $(N$ and $N,+1)$ categories. Five Ss totalled lowest under $\mathrm{N},+1$; the other under $\mathrm{N}$. Statistical significance obtained on the four comparisons of "high" conditions with "low" conditions ( $p$

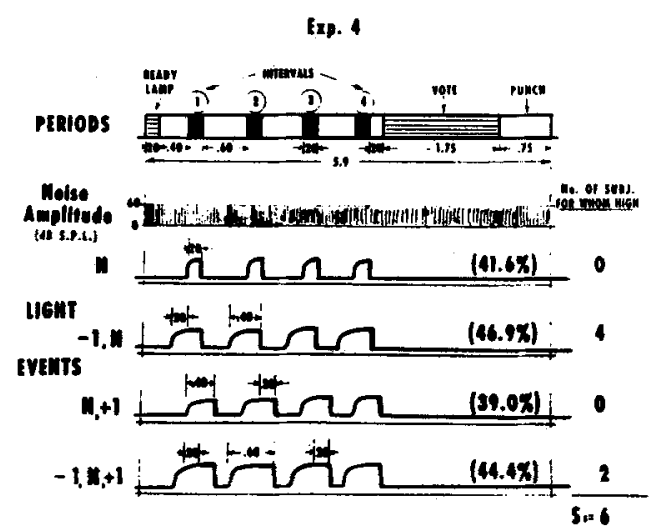

Fig. 7. Conditions and outcome information for Experiment 4. 


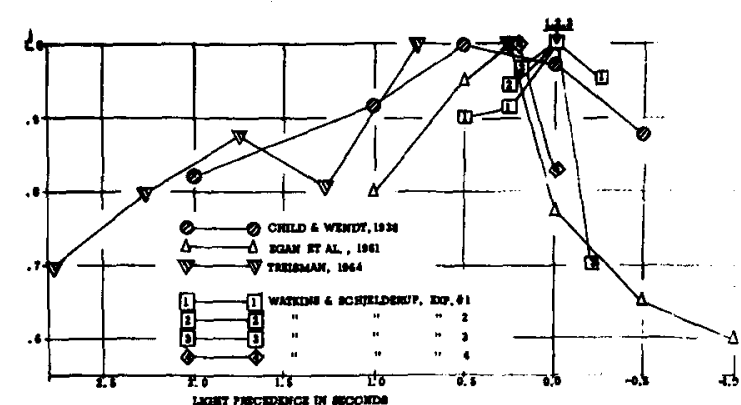

Fig. 8. Ordinate: proportional auditory achievement under particular light stimulus conditions, computed separately for each experiment, and normalized so that maximum mean $=1.0$. Abscissa: Onset time of photic stimulus in seconds relative to start of auditory observation periods.

$<.05)$. Within the categories, the differences were not statistically significant. 4

One of the high $(-1, N)$ and one of the low $(N)$ performance conditions had a common factor concelved as possibly of special value: concurrent cessation of light stimulus and possible signal interval. Condition $-1, N,+1$ did not have this characteristic but was, nevertheless, a rival of $-1, N$. Though small, the consistent performance decrements between $\mathrm{N}$ and $N,+1$, and between $-1, N$ and $-1, N,+1$ suggest reality of a degrading effect of delayed cessation of light stimuli relative to the tonal signal.

\section{Onsets}

\section{CONGLUSIONS}

Our forced-choice experimental series produced answers to questions regarding onset times and durations of useful light auxiliaries, which are only in partial agreement with the reported findings of experiments which employed other experimental procedures. Figure 8 is a graphic summarization of the results of three experiments cited early in this report; of the outcomes of Experiments 1, 2, and 3; and of the two .4 sec. durations of Experiment 4 .

A survey of the data given in Fig. 8 seems to indicate that a very desirable course of action for one interested in furnishing light stimuli to assist persons engaged in tone detection tasks would be to approximate the $-1, N$ condition of Experiment 4 . If confronted with a requirement, however, that light duration not exceed the length of the signal (when one is presented), note that light onset precedence showed no significant advantage over concurrent onset in the experiments reported. Additional experiments performed, but not formally reported, further support a belief in the essential equality of light stimuli of the classes "-1" and " $\mathrm{N}$ " in effectiveness as auxiliaries to tonal signal detection, in forced-choice trials of the sort used.

Although data are limited, the literature dealing with experiments other than forced-choice contains indications that onsets of light stimuli should precede acoustic stimuli by some amount of time, in order to contribute maximally to hearing. The results reported by Treisman, by Egan et al, and by Child and Wendt appear to converge toward optimal light onset precedence in the vicinity of $.3 \mathrm{sec}$.

Our forced-choice finding, in agreement with studies using other methods, was that a light stimulus delayed to the extent of having no overlap with possible signal intervals is relatively poor. Such comparisons with no-flash trials as can be made (Child \& Wendt; our Experiment 1) fail to justify affixing an "inhibitory" label on any of the light stimuli used. In our Experiment 3 it was as obvious as in much of the data of Egan et al that light events which occur after the intervals fall into an inferior class, for trials involving supposedly heavy dependence of Ss upon lights as cues for observation times.

\section{Cessations}

Two conditions used by Egan et al resembled our Conditions $-1, N$ and $N,+1$ of Experiment 4. In both investigations, a period of light onset precedence equal to signal duration, coupled with light persistence until termination of the interval (e.g., $-1, N$ ), ylelded highest average tone detectabilities of the entire experiment. However, in both experiments, light stimuli of equal duration but which commenced at the start of the intervals (e.g., $\mathrm{N},+1$ ) were clearly inferior to the conditions just mentioned. Egan et al have observed, in this connection,". . performance of a human listener declines when the observation interval and the alerting light are not coincident." (Egan et al, 1961, p. 780). Their data also warrant the more specific remark that performance declines when the cessation of a light of duration $.5 \mathrm{sec}$. Is not coincident with the end of an auditory observation period of .25 sec. The results of Experiment 4 conform, essentially, to the last statement, a small duration difference notwithstanding. Perhaps cessation relations are nearly as influential as onset relations in determining the effectiveness of light auxdliaries.

\section{References}

Child, 1. L., \& Wendt, G. R. The temporal course of the influence of visual stimulation upon the auditory threshold. J. exp. Psychol., 1938, 23, 109-127.

Egan, J. P., Schulman, A. I., \& Greenberg, G. Z. Memory for waveform and time uncertainty in auditory detection. J. Acoust. Soc. Amer., 1961, 33, 779-781.

Treisman, M. The effect of one stimulus on the threshold for another: an application of signal detectability theory. Brit. J. stat. Psychol., 1964, 17, 15-35.

Watkins, W. H. Effect of intensity changes in auxiliary stimuli on auditory and visual signal detection. USAF ESD-TR-66-121. Condensed version appears in Symposium 16, Detection of Signals, Moscow: XVIIIth Int. Cong. Psychol., 1966. Pp. 75-83.

Watkins, W. H. Photic facilitation of tonal signal detection in a forced-choice situation. Psychon. Sci., 1966, 6, 477-478.

\section{Notes}

1. This is Electronic Systems Divis ion Technical Report 66-512 of the Air Force Electronic Systems Division, Air Force Systems Command. This research was performed at the Decision Sciences 
Laboratory as part of Project 7682, Man-Computer Information Processing. Parts of this were contained in a paper "Tonal Signal Detection under Various Light Stimulation Conditions"'presented to the Acoustical Society of Amer., June, 1966. Further reproduction is authorized to satisfy the needs of the United States Government. 2. Lt. Col., United States Air Force. Address: Behavioral Sciences Laboratory, 6570th Aeromedical Research Laboratories, WrightPatterson AFB, Dayton, Ohio. 45433.
3. On 16 occasions among 672 runs comprising these experiments, runs were terminated short of programmed completion because of S's failure to respond within the allowed trial period but with enough completed trials $(>30)$ for acceptance.

4. F ratios were 3.8 and $3.1(1,5 \mathrm{df})$ for the within-high and withinlow comparisons.

(Accepted for publication March 17, 1967.) 